\title{
COMMENT
}

Check for updates

\section{Removing barriers and disparities in health: lessons from the COVID-19 pandemic}

Emily Sirotich $\mathbb{1 D}^{1}{ }^{\bowtie}$ and Jonathan S. Hausmann $\mathbb{B}^{2,3}$

The COVID-19 pandemic has compelled the global community to work together to understand SARS-CoV-2 and mitigate its effects, but it has also highlighted health disparities faced by people from minority racial or ethnic groups and other marginalized populations. International collaboration needs to be leveraged to address these disparities and inequities.

Marginalized populations ... have been overwhelmingly affected by COVID-19

'Department of Health Research Methods, Evidence, and Impact, McMaster University, Hamilton, ON, Canada

${ }^{2}$ Program in Rheumatology, Division of Immunology, Boston Children's Hospital, Boston, MA, USA

${ }^{3}$ Division of Rheumatology and Clinical Immunology, Beth Israel Deaconess Medical Center, Boston, MA, USA.

凶e-mail: sirotie@mcmaster.ca https://doi.org/10.1038/ s41584-020-00524-8
The COVID-19 pandemic has challenged the world's scientists and physicians to overcome geographical barriers and develop novel methods of prevention, testing and treatment to alleviate the widespread effects of the virus. Solutions to many of these issues are now being developed through international collaboration; however, the COVID-19 pandemic has also highlighted the existence of substantial inequities in health and in the delivery of health care. Marginalized populations, such as Indigenous communities, minority ethnic groups, people with disabilities and elderly individuals have been overwhelmingly affected by COVID-19 - not because of innate differences inherent to these groups, but because of multiple existing psychosocial, political, economic, occupational and nutritional factors ${ }^{1-3}$. The removal of structural barriers within societies and health-care systems needs to be prioritized to ensure equitable care for all people, not only during the COVID-19 pandemic but also beyond. In this Comment article, we identify and discuss ethnic and racial disparities in health care, under-representation of people from minority ethnic and racial groups in research, and highlight opportunities to address these issues through international collaboration.

\section{Barriers highlighted by the pandemic}

International reports indicate that rates of SARS-CoV-2 infection, hospitalization and mortality are disproportionately high in minority ethnic groups and low-income populations ${ }^{4}$. In the UK, people whose ethnicity is recorded as mixed, South Asian, Black or other are at increased risk of acquiring and dying from COVID-19, as compared with white people, even after adjusting for the presence of comorbidities ${ }^{2}$. Studies from the USA report similar findings, with Black, Latinx and Native American individuals being overrepresented among patients diagnosed with COVID-19 and having poorer outcomes than white individuals ${ }^{1}$. Black children and Hispanic and Latinx children are also more likely to develop multisystem inflammatory syndrome in children (MIS-C), a hyperinflammatory syndrome that follows SARS-CoV-2 infection, than the population in the USA overall $^{5}$. Nevertheless, while Black people have faced an increased burden of COVID-19, they have been systematically under-represented in epidemiological COVID-19 studies and clinical trials ${ }^{3}$, making it more difficult to generalize study results to the populations most affected.

In North America, members of minority racial and ethnic groups are disproportionately affected by poverty, the burden of ill health, limited access to healthy food, high-density housing and the need to work in 'essential' industries during the pandemic ${ }^{1}$. For example, a report by the New York City Comptroller of front-line workers in industries such as cleaning services, public transit, food and family services showed that over $75 \%$ were people of colour, over $50 \%$ were foreign-born and $8 \%$ were living at or below the federal poverty line during the COVID-19 pandemic ${ }^{6}$. The increased risk of exposure to SARS-CoV-2 experienced by front-line workers will also affect the populations disproportionately employed in these positions.

As we learn more about SARS-CoV-2, exposure is the main driver of infection. The ability to remain socially distant might not be equally available among all groups; those without financial means could be more reliant on public transportation, where social distancing is limited, and share housing with multiple people ${ }^{7}$.

\section{Lessons from SLE}

Rheumatologists might recognize the similarities between the health disparities of COVID-19 with those that affect people with systemic lupus erythematosus (SLE). As with COVID-19, SLE is more prevalent in minority racial or ethnic groups worldwide ${ }^{7}$. Outcomes also tend to be worse for Black people in the USA, who are more likely to develop lupus nephritis and have higher mortality rates than white people ${ }^{8}$. The reasons 


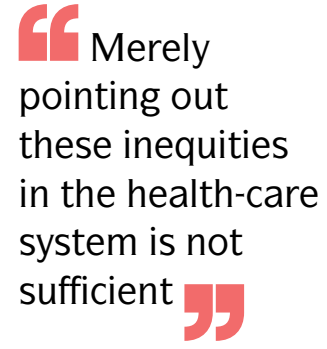

for these disparities in people with SLE include the social determinants of health such as environmental factors, psychosocial stress, adverse childhood experiences and poor access to care, among others 9 .

As with COVID-19, research studies of people with SLE continue to over-represent white participants while Black participants remain under-represented and understudied $^{7}$. Barriers to the enrolment of non-white participants in research studies include structural racism, historic mistreatment of people from under-represented groups in research studies, implicit bias of physicians and lack of trust of physicians owing to a historical legacy of mistreatment of vulnerable individuals ${ }^{8}$.

Fortunately, change is at hand. Researchers have begun to partner with community organizations and community-based social networks to enhance SLE education and awareness in the Black community ${ }^{10}$. The ACR has also received funding from the Office of Minority Health for Materials to Increase Minority Involvement in Clinical Trials (MIMICT) to increase the participation of minority groups in SLE clinical trials.

\section{International collaboration in research}

The COVID-19 Global Rheumatology Alliance (C19-GRA) was formed in March 2020 to study the global effects of the pandemic on people with rheumatic diseases. This initiative established a registry of physicianentered data on people with rheumatic diseases who develop COVID-19, as well as a patient experience survey of those with rheumatic diseases, with or without COVID-19. The goals of these projects are to better understand the effects of the pandemic on people with rheumatic diseases and explore factors that might affect their risk of COVID-19 and severe disease.

The C19-GRA patient survey was distributed in partnership with patient support organizations from across the world, translated into nine languages, and received participation from people in over 100 countries. However, individuals from certain racial and ethnic groups remain under-represented in the study. This disparity is most evident among respondents with SLE, as the SLE cohort in the C19-GRA patient survey is still primarily white (unpublished data).

Fortunately, the C19-GRA can learn from past challenges and other initiatives to pave a more inclusive way forward. In the next few months, the C19-GRA will develop a prospective registry of people with rheumatic diseases to study the short-term and long-term outcomes of the COVID-19 pandemic. We will continue working with patient partners to develop our survey instruments and recruitment materials, engage a more inclusive and representative cohort through partnership with community organizations involving minority racial and ethnic groups, and share our data back to these organizations to enhance the value of research participation. Also, we aim to collect more detailed measures of socioeconomic status, including type of housing, educational achievement, occupation and local environment to examine how these measures affect the development of COVID-19 and its related complications. We hope our research will identify areas amenable for intervention to enhance health and improve health equity.
Many other international collaborations have formed in response to the COVID-19 pandemic to better study how the disease affects vulnerable groups. For instance, the COVID-19 Sickle Cell Registry was established in the USA to capture international cases of COVID-19 that are occurring in children and adults living with sickle cell disease.

To take action and generate change, the C19-GRA, the ACR and corporate sponsors are prepared to invest in research to explore the effects of COVID-19 in vulnerable populations. The C19-GRA will collaborate with the International League of Associations for Rheumatology to provide grants to researchers and rheumatologists from countries that are under-represented in our projects, to support the enrolment of people with rheumatic diseases from these countries.

As we come together as a global community to understand and minimize the effects of COVID-19, we are encouraged to apply the lessons we have learnt about structural barriers in society to current research. Merely pointing out these inequities in the health-care system is not sufficient; we must enable action through dedicated funding and partnership to address them. The structural barriers in society will continue to disproportionately affect the most vulnerable groups until our world commits to abolishing the underlying problems within our systems.

1. Tai, D. B. G., Shah, A., Doubeni, C. A., Sia, I. G. \& Wieland, M. L. The disproportionate impact of COVID-19 on racial and ethnic minorities in the United States. Clin. Infect. Dis. https://doi.org/ 10.1093/cid/ciaa815 (2020).

2. Williamson, E. J. et al. Factors associated with COVID-19-related death using OpenSAFELY. Nature 584, 430-436 (2020).

3. Borno, H. T., Zhang, S. \& Gomez, S. COVID-19 disparities: An urgent call for race reporting and representation in clinical research. Contemp. Clin. Trials Commun. 19, 100630 (2020).

4. Abrams, E. M. \& Szefler, S. J. COVID-19 and the impact of social determinants of health. Lancet Respir. Med. 8, 659-661 (2020)

5. Feldstein, L. R. et al. Multisystem inflammatory syndrome in US children and adolescents. N. Engl. J. Med. 383, 334-346 (2020).

6. Stringer S. M. New York City's Frontline Workers https://comptroller. nyc. gov/wp-content/uploads/documents/Frontline Workers_032020. pdf (Office of the New York City Comptroller, 2020).

7. Yancy, C. W. COVID-19 and African Americans. JAMA 323 1891-1892 (2020).

8. Lima, K. et al. Factors associated with participation in rheumatic disease-related research among underrepresented populations: a qualitative systematic review. Arthritis Care Res. 72, 1481-1489 (2019).

9. DeQuattro, K. et al. Relationships between adverse childhood experiences and health status in systemic lupus erythematosus. Arthritis Care Res. 72, 525-533 (2020).

10. Feldman, C. H. \& Ramsey-Goldman, R. Widening disparities among patients with rheumatic diseases in the COVID-19 era: an urgent call to action. Arthritis Rheumatol. 72, 1409-1411 (2020).

Acknowledgements

E.S. and J.S.H are steering committee members of the COVID-19 Global Rheumatology Alliance (C19-GRA) and lead patient engagement and patient registries, respectively. The views expressed here are those of the authors and participating members of the C19-GRA and do not necessarily represent the views of the ACR, EULAR, or any other organization.

\section{Competing interests}

J.S.H. has received grants from the Rheumatology Research Foundation, Childhood Arthritis and Rheumatology Research Alliance (CARRA), and has acted as a consultant to Novartis, unrelated to this work. E.S. is a Board Member of the Canadian Arthritis Patient Alliance, a patient run, volunteer-based organization whose activities are primarily supported by independent grants from pharmaceutical companies. 